\title{
ON GROUP ALGEBRAS
}

BY MARTHA SMITH

Communicated by I. N. Herstein, March 13, 1970

For any (discrete) group $G$ and any field $F$, let $F G$ denote the group algebra of $G$ over $F$. Thus elements of $F G$ are finite formal sums $\sum a(g) g$, where $a(g) \in F, g \in G$.

THEOREM 1. Suppose $G$ is any group and $F$ is the field of complex numbers. Let $*$ denote the involution on $F G$ given by

$$
\left(\sum a(g) g\right)^{*}=\sum \overline{a(g)} g^{-1} \text {. }
$$

Let $Z$ denote the center of $F G$. Then there exists a function 4 from $F G$ into $Z$ with the following properties:

(i) $(a+b)^{\natural}=a^{\natural}+b^{\natural}$,

(ii) $(z a)^{\natural}=z a^{\natural}$ for $z \in Z$,

(iii) $(a b)^{\natural}=(b a)^{\natural}$,

(iv) $z^{\natural}=z$ for $z \in Z$,

(v) $\left(a^{*}\right)^{\natural}=\left(a^{\natural}\right)^{*}$,

(vi) $\left(a a^{*}\right)^{\natural}=0$ implies $a=0$.

In fact, if $W(G)$ is the $W^{*}$-algebra generated by the left action of $G$ on $l^{2}(G)$, then we may embed $F G$ in $W(G)$, and then the above function is just the restriction to $F G$ of the function $\xi$ on $W(G)$ studied by Dixmier [2].

A two-sided ideal of a ring $R$ is said to be an annihilator ideal of $R$ if it is the left annihilator of some subset of $R$.

Lemma 2. Let $F$ be a field of characteristic zero and $G$ any group. If $I$ is an annihilator ideal of $F G$ and $a, b$ are elements of $I$ such that $a x b$ $-b x a \in I$ for all $x \in F G$, then there exist elements $y, z$ in the center of $F G$, and not both zero, such that $y a-z b \in I$. If further $a(F G) b \Phi I$, then $y$ and $z$ may be chosen so that $y a \notin I$.

CoROLlaRy 3. If $F$ is algebraically closed of characteristic zero and $F G$ is an order in a ring $Q$, then the center of $F G$ is an order in the center of $Q$.

Given a group $G$, let $\Delta$ denote the subgroup of elements which have only finitely many conjugates in $G$. Let $\Delta^{+}$denote the subgroup of torsion elements of $\Delta$. By a result of Passman [5], $F G$ is semiprime

AMS Subject Classifications. Primary 1880; Secondary 1649, 1620, 1656, 4665.

Key Words and Phrases. Group algebra, annihilator ideal, polynomial identity, classical quotient ring, von Neumann regular ring, $W^{*}$-algebra, trace. 
if and only if $G$ has no finite normal subgroups of order divisible by the characteristic of $F$. By a result of Connell [1], $F G$ is prime if and only if $G$ has no finite normal subgroups.

THEOREM 4. If FG is semiprime and has a classical ring of quotients which is finite dimensional over its center, then $[G: \Delta]<\infty$.

From Theorem 4 and results of Procesi [7], Posner [6] and, Kaplansky [4] on polynomial identities, we obtain a strengthening of two special cases of a result of Isaacs and Passman [3].

Corollary 5. If $G$ has no finite normal subgroups and $G$ satisfies a polynomial identity of degree $n$, then $G$ has an abelian normal subgroup of finite index $\leqq[n / 2]^{2}$.

Corollary 6. If $G$ is finitely generated, $F G$ is semiprime, and $F G$ satisfies a polynomial identity, then $G$ has an abelian subgroup of finite index.

Also as an application of Lemma 2 we obtain

THEOREM 7. If $F \subset K$ are fields of arbitrary characteristic and $I$ is a prime ideal of $K G$ which contains no nonzero central elements, then $G$ has no finite normal subgroups and $I \cap F G \neq 0$.

As an outgrowth of the investigations of annihilator ideals and rings of quotients of group algebras, the following results were obtained.

THEOREM 8. Suppose FG is semiprime. Then if $I$ is any annihilator ideal of $F G, I=\left(I \cap F \Delta^{+}\right) F G$.

Corollary 9. Suppose $F G$ is semiprime, $I$ an annihilator ideal of $F G$, and $a \in I$. Then there exists a central idempotent $e \in I$ such that $a=e a$.

Corollary 10. Suppose FG is semiprime. Then the classical ring of quotients of the center of $F G$ is a von Neumann regular ring.

Proofs of these results will appear in the author's doctoral thesis.

The author wishes to thank Professor D. S. Passman for pointing out that the technique of Theorem 4 could be applied in characteristic not zero, and that the idempotent produced in Corollary 9 is central. Professor Passman has recently developed the author's approach to polynomial identities in group algebras in a combinatorial rather than ring-theoretic manner to extend the results of I. M. Isaacs and himself [3] to the general semiprime case. He has informed the author that he also has a proof of Theorem 8. 


\section{REFERENCES} \#3666.

1. I. Connell, On the group ring, Canad. J. Math. 15 (1963), 650-685. MR 27

2. J. Dixmier, Les anneaux d'operateurs de classe finie, Ann. Sci. École Norm. Sup. (3) 66 (1949), 209-261. MR 11, 370.

3. I. M. Isaacs and D. Passman, Groups with representations of bounded degree, Canad. J. Math. 16 (1964), 299-309. MR 29 \#4811.

4. I. Kaplansky, Rings with a polynomial identity, Bull. Amer. Math. Soc. 54 (1948), 575-580. MR 10, 7.

5. D. Passman, Nil ideals in group rings, Michigan Math. J. 9 (1962), 375-384. MR $26 \# 2470$.

6. E. Posner, Prime rings satisfying a polynomial identity, Proc. Amer. Math. Soc. 11 (1960), 180-183. MR 22 \#2626.

7. C. Procesi, On rings with polynomial identities, Thesis, University of Chicago, Chicago, Ill., 1966.

University of Chicago, Chicago, Illinois 60637 